Contribución especial

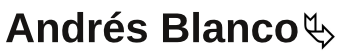

\section{Sobre la conveniencia de la regla fiscal Política fiscal y democracia}

\section{On the convenience of the fiscal rule. Fiscal policy and democracy}

\section{Sobre a convêniencia da regra fiscal. Política fiscal e democracia}

Resumen: La discusión acerca de la adopción de una regla fiscal (entendida como una restricción permanente al crecimiento del gasto público) en un tratados internacionales, la Constitución o la legislación ordinaria, debe partir del análisis de los efectos económicos que tiene una política de esa índole. La contención del gasto público es una propuesta de la corriente económica neoclásica, para la cual el gasto público tiene un efecto distorsionante, ya que supone que las personas lo interpretan como un aumento de la deuda pública y de los impuestos para solventar dichos incrementos. Por lo tanto, dicha corriente sostiene que la restricción al crecimiento del gasto público favorece la inversión y el consumo. Esta creencia es fuertemente contestada desde el poskeynesianismo, que considera al gasto público como un componente de la demanda que favorece el consumo y la inversión, y más específicamente como el único componente de la demanda que puede ser utilizado como herramienta contra las recesiones. Desde este último punto de vista, las reglas fiscales no serían deseables en absoluto, ya que limitan la capacidad de los gobiernos de adoptar políticas de combate a la recesión y el desempleo. Las evidencias empíricas comparadas de países con y sin reglas fiscales, así como también de la evolución del gasto público uruguayo, son tendencialmente contrarias a la conveniencia de las reglas fiscales, ya que éstas no evitaron las recesiones sino que, plausiblemente, tendieron a hacerlas más duraderas. Por otro lado, las evidencias también muestran que, contrariamente a lo sostenido por los defensores de las reglas fiscales, el incremento de la deuda pública es independiente del gasto público y el déficit. Por último, se concluye que las reglas fiscales son opuestas a una concepción consecuente de la democracia deliberativa, ya que sustrae a la

Dr. En Derecho y Ciencias Sociales. Profesor de Derecho Financiero, Facultad de Derecho, Universidad de la República. 
decisión democrática y al debate público un aspecto central de la vida social como lo es la política fiscal.

Palabras clave: regla fiscal, gasto público, política fiscal, deuda pública, déficit fiscal.

Abstract: The discussion on the adoption of fiscal rules (understood as permanent limitations of the growth of public spending) in the Constitution, international treaties or even ordinary legislation, must be conducted on the basis of their economic effects. The limitation of the growth of public spending is a central proposal of the neoclassical theory in economics, which believes that such spending have distorting effects since individuals interpret it as future increases of public debt and taxes, and consequently retract their investment and consumption. Therefore, neoclassical authors affirm that cuts in public spending stimulate private consumption and investment. That belief is hardly contested from the Postkeynesian theory, which considers public spending as a part of demand that favours consumption and investment, and more specifically as the unique part of demand that can be a tool against recessions and unemployment. From that perspective, fiscal rules are not advisable at all, because they limit the possibility of adopting policies of struggle against recession and unemployment. Empirical evidences from countries with and without fiscal rules, as well as from Uruguay, suggest that such rules have not stimulated consumption and investment and, on the contrary, contributed to extend temporarily the adverse effects of recessions. On other hand, there are robust evidences of the independence of public debt respecting public spending and deficit, in opposition to the neoclassical thesis. In a political perspective, in turn, fiscal rules are contrary to the conception of deliberative democracy, since they exclude from the democratic decision and public debate a central issue of social life like the fiscal policy.

Keywords: fiscal rule, public spending, fiscal policy, public debt, fiscal déficit

Resumo: A discussão com relação á incorporação de uma regra fiscal (entendida como uma restrição permanente ao incremento do gasto público) na Costituição, Tratados internacionais ou legislação nacional, deve partir do análise de uma política dese teor. A contenção do gasto público é uma proposta da teoría económica neoclásica, a qual mantém que o incremento do gasto público ten efectos distorcidos, porque as pessoas interpretam o incremento como um aumento futuro da dívida pública e os impostos. Portanto, para tal teoría a restrição ao crescimento do gasto público favorece o consumo e o investimento. Essa crença e fortemente contestada desde a teoría Poskeynesiana, qual acredita que o gasto público é um elemento da demanda que favorece o consumo e o investimento, e mais específicamente é um instrumento de combate contra as recessôes. Desde este ponto de vista a adopcão da regra fiscal é inconveniente porque limitam a capacidade do governo de combatir a recessão e o desemprego. As evidencias empíricas de países com e sem regras fiscais, e também do Uruguai, sugerem que tais regras não es- 
timularam o cosumo e o invetimento mas, ao contrario, contriuiron a prolongar os efectos adversos das recessôes. Alem disso, também as evidencias indicam a independencia da dívida pública do gasto público e do déficit. Desde uma perspectiva política, as regras fiscais são opostas a uma conceição consecuente da democracia deliberativa, ja que substrai da decissão democrática e do debate público um assunto central da vida social como a política fiscal.

Palavras-chave: regra fiscal, gasto público, política fiscal, dívida pública, déficit fiscal. 


\section{Introducción}

Las reglas fiscales han llegado a Uruguay, y junto con ellas el debate en torno a su conveniencia, tal como ha sucedido desde hace un tiempo en Europa y otros países de América Latina. La ley Nro. 19.889 (Ley de Urgente Consideración) prevé, en sus artículos 209 a 212, una "regla fiscal programática", en tanto encomienda a las leyes de presupuesto limitar el incremento del gasto público en función del "resultado fiscal estructural” estimado por el Ministerio de Economía y Finanzas. Luego, el artículo 7 de la ley Nro. 19.924 (Ley de Presupuesto del período 2020-2024) facultó al Poder Ejecutivo a ajustar las asignaciones presupuestales de la mayoría de los organismos comprendidos en el presupuesto nacional a fin de cumplir con la meta fijada por la Ley de Urgente Consideración. Esta última autorización torna pues operativa la regla fiscal prevista en la Ley de Urgente Consideración, ya que la corrección de las asignaciones de gasto implica la aplicación de la regla de contención del crecimiento del mismo. De cualquier forma la regla fiscal se convierte en una herramienta discrecional u optativa, ya que el Poder Ejecutivo está autorizado, pero no obligado, a aplicar dicho ajuste.

En el campo académico, Risso Ferrand ha defendido la conveniencia de las reglas fiscales y su incorporación a las Constituciones, propuesta que incluye a Uruguay dado que la Constitución vigente de este país no contiene hoy una restricción de ese tipo (Risso Ferrand, 2020).

El momento es, pues, el indicado para debatir en toda su extensión sobre este tipo de normas, y en particular sobre la conveniencia de su adopción por las leyes, los tratados y -especialmente- la Constitución. En este artículo sostendré que las reglas fiscales no son convenientes, por dos grupos de motivos:

- Desde el punto de vista económico los supuestos teóricos en los que se basan las reglas fiscales son erróneos, lo cual se confirma con la experiencia de aplicación de dichas reglas en algunos países que las adoptaron, en donde no se cumplieron los efectos que predecían sus defensores. 
- Desde el punto de vista de la organización política, las reglas fiscales significan una restricción al funcionamiento democrático del Estado, ya que sustraen en un grado importante, tanto al debate como a la decisión del parlamento, un aspecto medular de la actividad estatal como lo es el gasto público.

Primeramente, haré alguna reflexión acerca de la necesidad de que el discurso académico de los juristas se ocupe de los supuestos de hecho sobre los que apoya sus opiniones, incluyendo la discusión teórica sobre problemas que estrictamente no son "jurídicos” (aunque sí normativos), como el modelo de toma de decisiones del Estado. En segundo lugar, analizaré las tesis de una corriente de la teoría económica que fundamentan la conveniencia de regla fiscal. Dicho análisis se hará en dos niveles: primero se analizará la consistencia de esa tesis y se expondrá también la tesis teórica rival, para la cual no es conveniente la regla fiscal, y luego se contrastarán ambas teorías con datos estadísticos tanto del extranjero como de Uruguay. En tercer lugar, se analizarán los argumentos políticos favorables y contrarios a la regla fiscal.

\section{Algunos apuntes sobre el diseño de políticas y los supuestos teó- ricos en el discurso académico de los juristas}

La proposición y objeción de la regla fiscal podría parecer una cuestión ajena al objeto tradicional de la dogmática jurídica, la que, al menos en los países que forman parte de lo que podríamos llamar "cultura jurídica europeo-continental” (como lo somos los países latinoamericanos), suele concebirse como un discurso orientado fundamentalmente a los juristas prácticos: abogados, jueces, administradores, etc..

Esa concepción estrecha de la dogmática no es, sin embargo, necesaria ni conveniente. La dogmática es un discurso normativo inclusive cuando se limita -aparentemente- a "interpretar" textos ya dados, como una ley o la Constitución, ya que los supuestos "significados" que resultan de esa labor no son meros desarrollos del texto original, sino auténticos textos normativos nuevos que se adicionan a la Constitución, la ley, etc. De allí a proponer cambios a los propios textos constitucionales, legales o reglamentarios no hay más que un paso, y por cierto que históricamente muchos dogmáticos lo han dado, de modo tal que puede decirse que una de las funciones de la dogmática es la proposición de soluciones de lege ferenda (Courtis, 2006, 126-127). 
Esa función de la dogmática la hace coincidir con la política entendida como la propuesta y diseño de soluciones normativas para la vida social, pero ello no es algo alarmante ni original, ya que hasta la más trivial labor dogmática tiene un papel político, en la medida en que se enfoca a conformar el entorno de decisión de órganos del Estado respecto de conflictos sociales, como lo son los órganos jurisdiccionales.

Ahora bien, la admisión de que la dogmática tiene un contenido político, muy visible cuando propone reformas legislativas o constitucionales, implica admitir el uso de argumentos y conceptos de la teoría política para admitir o rechazar una propuesta dogmática. Esto es especialmente relevante en la discusión sobre la conveniencia de las reglas fiscales, ya que ella involucra cuestiones de diseño institucional y de organización del Estado desarrolladas, precisamente, en el ámbito de la teoría política. Por lo tanto, al debatir sobre la regla fiscal será no sólo posible, sino necesario, confrontar argumentos que, a diferencia de los razonamientos tradicionales de la dogmática, no imaginan ninguna "norma jurídica”, ni siquiera como "principios” o "derechos”, como su fundamento, sino que directamente miran el funcionamiento del Estado como un fenómeno social. Desde luego que ello nos conduce a borrar la frontera entre lo "jurídico” y lo "político”, pero no es pertinente ahondar en esta ocasión sobre dicho tópico.

Lo anterior me lleva a otro punto importante: para discutir acerca de la conveniencia de la regla fiscal es necesario asumir una cierta descripción de la economía, o más precisamente una visión teórica de la economía capitalista. En efecto, y si bien la dogmática, la política y cualquier discurso normativo tienen propiedades distintas de los lenguajes descriptivos, está claro que cualquier discurso normativo racional debe asumir alguna descripción de la vida social, al menos desde dos puntos de vista: a) para identificar el entorno fáctico que se toma como supuesto; y b) para prever los efectos de las soluciones que se proponen (Nino, 1993, 36-37; Sarlo, 2006, 197-200).

Es posible que a este respecto se asuman, sin discutirlas, ciertas premisas de una disciplina teórica sobre las cuales se construye una propuesta normativa, como lo hace Risso Ferrand con algunas hipótesis de la teoría económica neoclásica en las que se asienta la regla fiscal (Risso Ferrand 2000, 4). Pero también es posible generar aquí y ahora esa discusión teórica, lo que equivale, en nuestro caso, a debatir acerca de las propiedades del gasto público y los tributos desde el punto de vista económico. Este punto me parece crucial, porque es el verdadero corazón de la discusión: esta es una disputa entre diferentes concepciones de económicas, por lo que si no las discutimos como tales estaremos debatiendo sólo aspectos laterales. 


\section{Concepto de "regla fiscal"}

Primeramente definamos con mayor precisión qué entendemos por "regla fiscal”, reiterando que hay dos grandes grupos de las mismas (Kopits y Symansky, 1998, 2-4).

Un primer grupo de reglas fiscales, menos frecuente en la literatura y en la práctica legislativa, pero ya vigente en Uruguay, consiste en la limitación directa de la deuda pública, sea estableciendo directamente un monto máximo de endeudamiento, o en comparación con otro parámetro económico, como el producto bruto. A este conjunto de reglas pertenece la ley Nro. 17.947 de Uruguay, la cual establece que, a partir del año 2009, el Poder Ejecutivo podrá emitir deuda pública con el límite de US\$ 250 millones de incremento respecto del volumen de la deuda del año anterior, con un margen adicional de hasta un $50 \%$ por circunstancias extraordinarias. Con todo, esta regla fiscal orientada a limitar el endeudamiento carece de rigidez, ya que al estar fijada en una simple ley puede ser modificada por otra simple ley en cualquier momento, además de que no se establece ninguna sanción para el caso en que se exceda el límite.

El segundo grupo de reglas fiscales, que es el más frecuente, consiste en una limitación del gasto público y/o el déficit fiscal (es decir, la diferencia entre el gasto público y los ingresos públicos). El límite puede consistir en la directa prohibición del déficit, como lo hace el artículo 225 de la Constitución uruguaya respecto de los presupuestos de los Gobiernos Departamentales. Lo más habitual, sin embargo, es que las reglas fiscales limiten el incremento del gasto público o el nivel del déficit público en función de una estimación de la evolución futura de una variable económica, generalmente el Producto Bruto Interno (PBI).

La variante más sencilla de esta clase de regla fiscal es vincular el déficit con una estimación de la evolución del PBI, como es el caso de la Unión Europea con sus sucesivos "Pactos de Estabilidad Fiscal y Crecimiento" (Larch \& Jonung, 2014). En estos casos se establece que el déficit no podrá superar un cierto porcentaje (por ejemplo el $3 \%$ en el caso europeo) del PBI del año siguiente, a cuyos efectos se faculta a algún órgano institucional para estimar cuál será el PBI futuro.

Otra variante, más sofisticada, de reglas fiscales de este tipo, es la propuesta en la Ley de Urgente Consideración en Uruguay y presente en otros países (por ejemplo Holanda), en las cuales el gasto público debe ajustarse al "resultado fiscal es- 
tructural”. Muy simplificadamente, estas reglas requieren que se estime para el período siguiente la variación del PBI eliminando los efectos del ciclo económico (es decir, los auges y recesiones que determinan alzas y caídas del PBI), y estimando ingresos tributarios también sin tener en cuenta las variaciones de la recaudación que derivan del ciclo económico. Sobre esas bases se establece cuál sería el gasto máximo que podría realizarse para que el déficit no superase un cierto porcentaje de esa estimación del PBI (Morales y Posada, 2010, 16-18).

En cualquiera de estas variantes, la regla fiscal se ejecuta mediante reducciones o ampliaciones (generalmente lo primero) de las asignaciones de dinero en favor de los órganos del Estado comprendidos en el Presupuesto, hasta alcanzar el nivel general establecido. Por ejemplo, en el caso de Uruguay el artículo 7 de la ley 19.924 establece cuáles Ministerios, entes y servicios verán reducidas sus partidas si el Poder Ejecutivo decide cumplir con la meta de resultado fiscal estructural de acuerdo con la Ley de Urgente Consideración.

Este artículo se focalizará en este segundo grupo de reglas fiscales orientadas a contener el incremento del gasto público.

\section{Supuestos teóricos de las reglas fiscales que limitan el gasto pú- blico y discusión conceptual de los mismos}

Sin entrar en mayores honduras, es muy visible que las reglas fiscales orientadas a contener el crecimiento del gasto público miran con desconfianza a este último, como un factor peligroso y negativo en el funcionamiento de la economía. Creo que es pertinente, pues, remontarnos a las bases más elementales de la teoría que sostiene la regla fiscal, para discutir integralmente toda su estructura conceptual.

La regla fiscal es uno de los eslabones finales de la teoría neoclásica (a veces también llamada marginalista por referencia a su uso del concepto de "utilidad marginal”), que es la concepción dominante del pensamiento económico actual tanto en lo académico como en lo político. Uno de los conceptos centrales de esta teoría es la "hipótesis de equilibrio": si la economía capitalista funcionara solamente en base a intercambios de mercancías y dinero, sin intervención del Estado ni otro agente supraindividual, se llegaría a un punto de estabilidad total en la producción, los precios y los costos (Walras, 1987, 279; Arrow, 1974). La estabilidad se produciría porque las personas que viven en estas sociedades son "sujetos racionales”, que adoptan sus decisiones en materia económica analizando todas las opcio- 
nes que tienen en cuanto a consumir, trabajar, invertir, etc., y eligiendo sólo aquella que les reporta mayor utilidad. Desde ese punto de vista, los ciclos económicos con sus sucesivas fases de crecimiento, estancamiento y recesión, obedecen a fallas de información o incertidumbres que hacen que los sujetos no puedan vislumbrar los efectos de sus decisiones (Lucas, 1975).

En este marco, tanto el gasto público como los tributos son elementos distorsionantes de las decisiones de las personas. En primer lugar, para esta teoría el Estado es un intruso en el funcionamiento económico, ya que tanto cuando establece regulaciones como cuando gasta y recauda, introduce en el funcionamiento de la economía factores ajenos a las puras decisiones individuales. Ese carácter distorsivo del gasto público y los tributos se acrecienta cuando la política fiscal es fluctuante, es decir cuando el Estado utiliza activamente el gasto público y los tributos para lograr determinados objetivos (Barro, 1981).

En este punto ya podemos entender el motivo por el que se proponen las reglas fiscales que limitan el crecimiento del gasto público (para los párrafos siguientes: Giavazzi \& Pagano, 1990; Bertola \& Drazen, 1991; Alesina \& Perotti, 1994). Asumiendo como irreal una hipótesis de ausencia de gasto público y tributos, ya que ello implica una desaparición del Estado que es fácticamente imposible incluso para versiones extremas de esta teoría, en todo caso es preciso -se nos dice- limitar todo cuanto se pueda la variación de dichos elementos.

En una situación en la que no hay ninguna limitación normativa a la modificación del gasto público, es obvio que éste puede elevarse indefinidamente, o bruscamente, en la medida en que así lo decida el gobierno dentro de lo permitido por la ley presupuestal del país de que se trate Ahora bien, para la corriente neoclásica los incrementos del gasto público se financian con tributos, o en su defecto con deuda pública, la que a la larga también se deberá cancelar con el producido por la recaudación tributaria. Los tributos reducen la capacidad de consumo y de inversión ya que disminuyen las dotaciones de dinero de las personas. Por lo tanto, dice esta corriente de pensamiento, los “sujetos racionales” interpretan el incremento del gasto público no sólo como una modificación del mismo, sino que advierten sus consecuencias mediatas de incremento de la deuda pública y los tributos, y reaccionan como si el aumento de estos últimos fuera un efecto presente. Es decir, para esta corriente los sujetos, a la vista del aumento del gasto público, reducen ahora el consumo y la inversión, sin esperar al incremento futuro de la deuda ni -sobre todo- el incremento futuro de los tributos. 
Para evitar esos efectos, nos dice esta corriente, el gasto público, la deuda pública y los tributos deberían moverse en forma lenta, pausada y previsible tanto en el corto, como en el mediano y largo plazo. En ese escenario, las personas (que, recordemos, son imaginados como "sujetos racionales”) interpretarán la estabilidad de esos tres elementos como una ausencia de incrementos futuros tanto del gasto como de los tributos, y a fortiori de la deuda pública como paso intermedio entre unos y otros. De esta forma, se reduciría la incertidumbre y se volvería a tomar las decisiones de consumo e inversión sólo en función de la utilidad que les proporcionan. Es más: el inicio de las políticas estabilizadoras del gasto público debería consistir en reducciones sustanciales del gasto público, especialmente en sus partes socialmente más sensibles (salarios, jubilaciones, subsidios, etc.), puesto que la disposición de un gobierno a adoptar medidas impopulares refuerza la convicción general de que se ha tomado en serio la estabilidad de las finanzas públicas.

Esto supone una restricción severa de la política fiscal activa, tanto en materia de gasto como de tributos, sin importar los objetivos concretos que se busquen (salir de una crisis económica, redistribuir el ingreso, disminuir la pobreza, etc.). Esto no quiere decir que esta versión de la teoría neoclásica condene irremediablemente el uso de las finanzas públicas para el logro de esos objetivos, pero sí que esas políticas deben subordinarse a la "estabilidad a la baja” del gasto público y la recaudación tributaria.

La mejor forma de mantener el gasto público contenido, impidiendo su aumento y su utilización como herramienta activa para llevar a cabo políticas públicas, es incluir en la Constitución o un Tratado internacional una prohibición de aumentar el gasto por encima de alguna pauta preestablecida. La preferencia por dar rango constitucional o de Tratado a esa limitación responde al deseo de ponerla a resguardo de las variaciones en la composición del parlamento, pero en última instancia su consagración a nivel meramente legal es preferible a su ausencia, para los defensores de este punto de vista. Esta es, en definitiva, la regla fiscal y su fundamentación.

Desde el punto de vista conceptual, el desarrollo teórico que lleva a proponer la regla fiscal merece varias objeciones. La primera es que esta corriente supone que el gasto público cae en una suerte de "agujero negro", ya que cuando se mira el funcionamiento de la economía desde el sector privado, parece que el Estado sólo detrae recursos mediante la recaudación, y que nadie se beneficia del gasto público. Pongo un ejemplo. Bertola y Drazen (en el trabajo citado más arriba) formalizan mediante una ecuación la "incertidumbre" generada por el gasto público y los tributos, en la que el consumo privado es determinado por los siguientes elementos: la 
utilidad que le reporta a la persona un cierto bien o servicio; sus ingresos (utilidades empresarias, salarios, etc.), que se suponen fijos; y por los tributos, que inciden negativamente porque merman los recursos que destina al consumo. Ahora bien, es evidente que el gasto público es -valga la redundancia- un gasto sólo desde el punto de vista del Estado, porque desde el punto de vista del sujeto que se beneficia del mismo, es un ingreso. Por ejemplo, el pago de un salario público es un gasto para el Estado, pero para el funcionario es un ingreso. A ello habría que agregarle que esos sujetos para quienes el gasto público es un ingreso, consumen. Por lo tanto, ¿por qué en una ecuación que expresa los factores que determinan el consumo se toman en cuenta sólo a los tributos, y no al gasto público, que evidentemente incrementa los ingresos de las personas privadas? Dicho de otro modo: si por un lado se toma en cuenta el efecto negativo de los tributos sobre los ingresos de las personas, debería también tomarse en cuenta el efectivo positivo del gasto público sobre dicho ingreso.

Otra objeción conceptual que se puede hacer al argumento neoclásico a favor de la regla fiscal, es la suposición de que las personas son "sujetos racionales". En realidad esta suposición entraña dos hipótesis, ambas sumamente cuestionables: a) que la única "racionalidad económica" es la derivada de la evaluación de utilidades individuales, y por lo tanto que las personas no tienen otras motivaciones para consumir, invertir, trabajar, etc.; y b) que las decisiones en materia económica se adoptan luego de una evaluación exhaustiva de toda la información disponible.

La primera hipótesis ha sido controvertida, entre otros, por Sen, quien ha señalado que las motivaciones de la conducta humana son tan múltiples y variadas en todos los ámbitos, que suponer que en el ámbito económico todas esas motivaciones se reducen a sólo una, no es en absoluto sensato. Más concretamente, Sen señala que es normal que las personas adopten una conducta por razones de "agencia", es decir, que la decisión se incline por lo que se considera bueno, aunque ello no signifique ninguna ventaja monetaria (Sen, 1999, 58 y ss.). Si la respuesta a esas objeciones consistiera en extender el concepto de "utilidad" a esas motivaciones diferentes a una ventaja económica, entonces el concepto de utilidad pasa a representar todo, lo que es igual a decir que no representa nada.

La hipótesis de que las decisiones, como regla, son tomadas en función de toda la información existente, es fácilmente contestable, la información disponible es inhumanamente vasta, por lo que no tiene sentido razonar en torno a una hipótesis imposible (Simon, 1959). Entiendo que esta última objeción es decisiva en contra del esquema explicativo propuesto por los economistas neoclásicos respecto del 
gasto público y la regla fiscal. En efecto, no es para nada realista suponer que los sujetos económicos permanentemente anticipan, quizás con años de distancia, que el gasto y/o la deuda pública de hoy se traducirán en aumentos tributarios y que, además, esos aumentos tributarios les perjudicarán. Todavía más irreal es suponer que ese tipo de anticipación es realizada por trabajadores, jubilados y pensionistas, que son quienes realizan el consumo final de bienes y servicios: es difícil pensar que las decisiones de esas personas de aumentar o disminuir el consumo se adopten luego de monitorear el estado actual y la proyección de evolución del déficit público.

Una crítica más específica a los proponentes de la regla fiscal apunta al silencio que los mismos guardan acerca de otros elementos que podrían ser más importantes que los aumentos eventuales de tributos a la hora de determinar la evolución de la inversión y el consumo. Así, sin abandonar una explicación neoclásica y sin dejar de proponer la regla fiscal, Fanelli ha señalado que el diseño de las reglas fiscales suele ignorar el impacto que tienen en América Latina los choques externos, lo cual impone, como mínimo, una atención a los mismos para proponer soluciones en materia de gasto público (Fanelli, 2011).

Las anteriores son objeciones al marco teórico general, y a las hipótesis particulares, que sostienen a la regla fiscal. Veamos seguidamente otras visiones que, ya desde fuera del marco teórico neoclásico, sostienen exactamente lo opuesto: la peligrosidad de establecer límites al gasto público y de reducir el margen de acción de la política fiscal.

\section{Supuestos teóricos adversos a la regla fiscal}

Si bien el pensamiento económico neoclásico ha sido totalmente dominante durante más de medio siglo, en simultáneo siguió desarrollándose -entre otras- la corriente económica que se ha dado en llamar "poskeynesiana”, que no comparte ni los supuestos teóricos ni las recomendaciones políticas de la corriente neoclásica.

En primer lugar, el poskeynesianismo descarta la hipótesis de equilibrio: la economía capitalista naturalmente tiende a los ciclos económicos con períodos de auge, estancamiento y recesión, los cuales no son consecuencias de "fallas en la información” o "fallas de mercado", sino consecuencias del funcionamiento normal de este tipo de economía. Esta hipótesis se construye, en primer lugar, a partir de la observación del desempeño histórico de las economías, la que revela que rara vez o nunca se está en situación de equilibrio y, en su lugar, que todos los países y regio- 
nes se desenvuelven invariablemente con subas y bajas, a veces bruscas, de la producción y el empleo. Esta característica se explica porque, para esta corriente, el factor determinante de la evolución de la economía capitalista es la demanda, pública y privada, de empresas y de consumo final, por lo que las oscilaciones de la misma permanentemente empujan al alza y la baja la producción y, detrás de ella, el empleo, entre otras variables (Keynes, 2018, 279 y ss.; Kalecki, 1971, 1-14 y 124-137).

La “demanda” incluye a la inversión (es decir, a la adquisición por las empresas de bienes duraderos destinados a la producción), el consumo de bienes terminados, las compras de sujetos del exterior (exportaciones) y las compras del Estado. Como se observa, en principio los componentes de la demanda operan de un modo no controlable, ya que involucran comportamientos colectivos que se desenvuelven a través de procesos sociales complejos, salvo uno: aquellos factores que dependen del Estado. Ello incluye tanto a las compras del Estado, como también al consumo de personas que reciben pagos del Estado (trabajadores públicos, jubilados, beneficiarios de subsidios, etc.). Por consiguiente, el gasto público es la herramienta por excelencia que tiene el Estado para revertir las crisis, cuando ya se han instalado, o para prevenirlas, cuando aún no se han producido (Kalecki, 1971, 15 y ss.; Keynes, 2018, 289).

Se puede ver entonces que para esta corriente de pensamiento el gasto público no es una interferencia causante de anomalías en el funcionamiento económico, sino un elemento que opera positivamente en este último. Como, por otro lado, en esta corriente no se supone a la sociedad como un conjunto de personas que deciden en función de toda la información, extrayendo conclusiones a mediano plazo (como lo hacen los neoclásicos), no se sostiene tampoco que las personas interpretan los aumentos de gasto público como aumentos futuros de tributos. Es decir, para los poskeynesianos no hay retracción del consumo debido a una "interpretación" a mediano plazo de los efectos del gasto público, sino que éste tiene un efecto directo y simple de incrementar la demanda.

A ese último respecto, cabe agregar que para un sector (no para la totalidad) de la corriente poskeynesiana, denominado "teoría del circuito monetario", el hecho de que el gasto público se efectúe en dinero y de que el Estado tenga el poder de emitirlo, implica que el gasto público no está restringido ni por la recaudación tributaria ni por los fondos obtenidos mediante la emisión de deuda pública. Eso no significa que los tributos no tengan una función esencial, ya que mediante su recaudación el Estado retira permanentemente dinero circulante que, de otra forma, provocaría un aumento incesante de precios. No obstante, esa idea lleva a sostener que 
el nivel del gasto público no está restringido por el nivel de recaudación, con lo cual el déficit público deja de ser una anomalía (Bell, 2001, 155; Parguez, 2001).

El desarrollo anterior no sólo justifica los incrementos del gasto público, sino que también lleva a afirmar que las reducciones del gasto público, así como su estabilización dentro de un ritmo contenido y previsible, como proponen las reglas fiscales, no son políticas deseables, sino todo lo contrario. Las reducciones o contenciones del gasto público suponen una contracción de la demanda, que acercan o agravan las crisis económicas, por lo cual las reglas fiscales eliminan el principal elemento con el que los gobiernos para combatir las recesiones económicas (Arestis \& Sawyer, 2004).

La objeción tradicional que se hace desde la corriente neoclásica a las hipótesis poskeynesianas consiste en afirmar que cuando el incremento del gasto público se convierte en una política fiscal constante, los sujetos anticipan ese ritmo y por lo tanto ya no reaccionan a los aumentos de gasto público utilizando esos fondos adicionales para consumir o invertir más. Al contrario, se sostiene que los incrementos del gasto público son interpretados como antesalas de futuras reducciones del mismo o aumentos de tributos (Alesina \& Perotti, 1996, 4).

La objeción recién vista debe dirimirse en el campo de las evidencias empíricas. En lo personal, aunque ya adelanté que en general me parecen convincentes las hipótesis poskeynesianas, creo que éstas tienen un límite, cual es la capacidad instalada y la estructura productiva de una economía. En efecto, las estructuras productivas de los países son previas a los factores de los que se ocupa la teoría poskeynesiana estándar, como también lo son a las hipótesis neoclásicas. Por lo tanto, la corrección de las aseveraciones poskeynesianas son directamente proporcionales a la madurez del desarrollo de una economía. No obstante, los países latinoamericanos tienen un grado de madurez mediano, por lo cual pueden ser un ámbito en el que podrían cumplirse las hipótesis de una y otra teoría. Veamos pues si hay evidencias que ayuden a resolver la discusión, y con ello arrojen luz sobre la conveniencia de la regla fiscal.

\section{Las evidencias empíricas}

Expuestas las tesis contrapuestas, veamos cuáles son las evidencias empíricas. Antes de pasar a la exposición de datos, es menester realizar dos aclaraciones. En primer lugar, adoptaré el criterio de Popper para evaluar los datos fácticos: si bien las evidencias coincidentes con una teoría tienen un papel importante ya que ratifi- 
can su poder explicativo, el papel fundamental lo cumplen las evidencias contrarias a una teoría en aspectos cruciales, ya que si bien nunca podemos dar por definitivamente confirmada una hipótesis, sí podemos y debemos darla por refutada cuando yerra en su pronóstico (Popper, 1994, 309 y ss.).

En segundo lugar, hay que señalar que, al menos en las ciencias sociales, los datos fácticos nunca son neutrales, ya que los propios "hechos" medidos, así como la recolección de evidencias y su interpretación, sólo son posibles si previamente se ha aceptado un paradigma teórico, por lo que es preciso mantener una gran cautela respecto de su fuerza explicativa. Por ejemplo, seguidamente se compararán datos estadísticos sobre el producto bruto y ciertas variables fiscales, por cuanto tanto la corriente neoclásica como el poskeynesianismo se interesan en el producto, particularmente en su crecimiento. Si, en cambio, adoptáramos -por citar sólo un ejemplo- una perspectiva ecológica de la economía, probablemente no existiría una preocupación por el crecimiento del producto bruto sino por otras variables.

Hechas las aclaraciones, observemos lo ocurrido con la Unión Europea, que -como indicamos más arriba- tiene desde 1997 una regla fiscal que limita el crecimiento del gasto público. Para una mejor comprensión se muestran conjuntamente las evoluciones del gasto público, el déficit público, la deuda pública y el producto bruto.

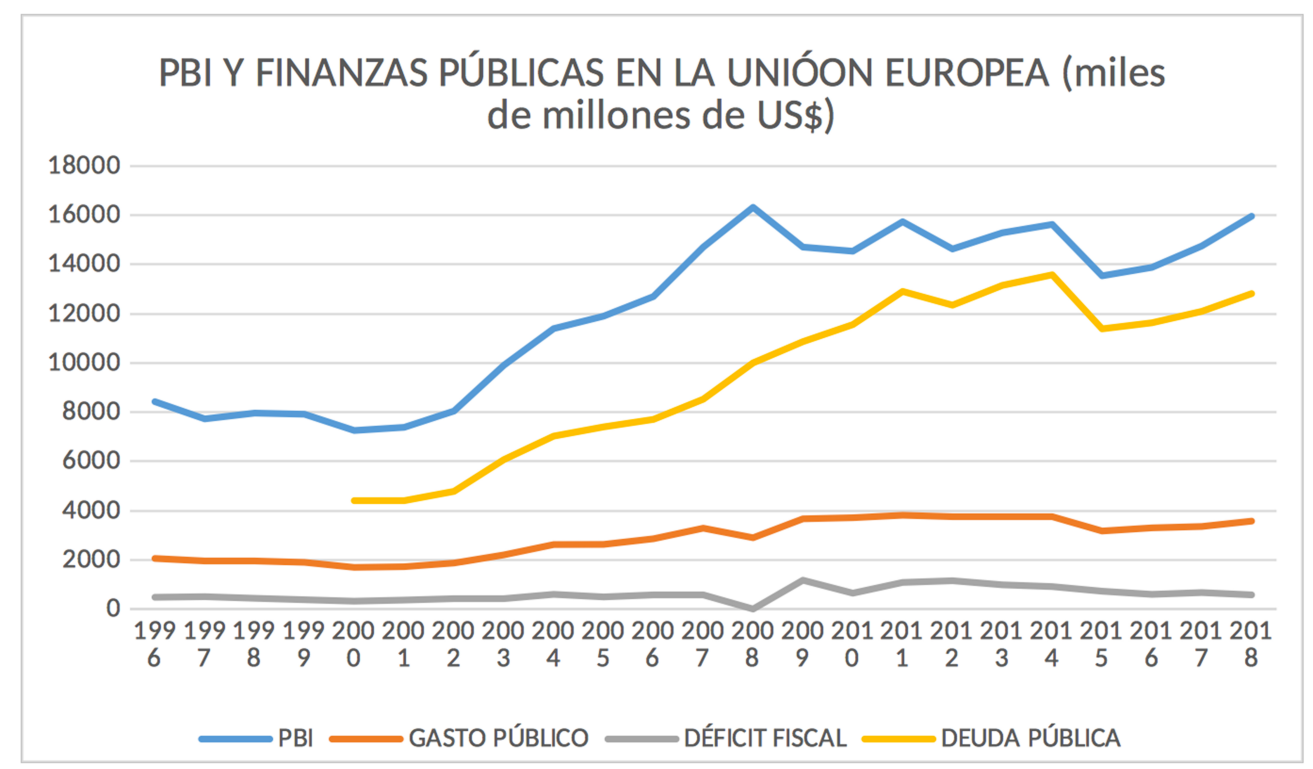

Fuente: elaboración propia a partir de datos del Banco Mundial, https://datos.bancomundial.org/indicator/ 
Si consideramos los datos del gasto público y el déficit, observamos que efectivamente la regla fiscal logró su objetivo directo, que era contener el crecimiento del gasto público y -sobre todo- del déficit fiscal: si bien en la primera década del siglo el gasto público europeo se duplicó, el déficit permaneció, en términos generales, igual. Ahora bien, este dato no significa gran cosa porque es tautológico: si hay una regla que dice que el gasto público debe crecer poco, y efectivamente el gasto crece poco, lo único claro es que se ha respetado la regla. Lo importante es observar si la regla fiscal tuvo el efecto de impulsar el consumo y la inversión, y con ello mejorar la evolución del producto bruto.

En ese sentido, se observa que en los ocho primeros años del siglo el producto bruto europeo tuvo un crecimiento impresionante que lo llevó a duplicarse, en simultáneo a la vigencia de la regla fiscal. En 2008, sin embargo, se produce una crisis que lo hace caer, teniendo un leve repunte seguido de otra recesión, un segundo repunte y una tercera recesión en 2015 y 2016, más profunda que las dos anteriores. Recién en 2018 se recupera el nivel del producto previo a la crisis de 2008, por lo que podemos decir que Europa tuvo una década de recesión y estancamiento. La conclusión obvia es que la regla fiscal no evitó la primera crisis, ni tampoco las que siguieron como secuelas de la primera. En otras palabras, en Europa coincidieron la "estabilidad fiscal", con el auge, la crisis y el estancamiento. Esto sugiera que la regla fiscal y su cumplimiento no provocan la estabilidad económica, y mucho menos el crecimiento.

Es más: si observamos en detalle la gráfica, advertiremos que la gran crisis europea de 2008-2009 fue precedida de una reducción del gasto público y de una disminución del déficit que llevó este último prácticamente a 0, lo cual es exactamente lo contrario a lo pronosticado por la corriente neoclásica. Por otro lado, las sucesivas crisis europeas en la década siguiente son acompañadas de una reducción del gasto público, lo cual puede atribuirse a la operatividad de la regla fiscal. Ambas comprobaciones demuestran que la regla fiscal implica la anulación de la política fiscal: cuando ella rige, los gobiernos, en principio, son mayormente espectadores del ciclo económico antes que agentes que combatan las crisis.

La evolución de la deuda pública en Europa también parece ir en contra de las hipótesis neoclásicas. Se puede observar que la deuda pública de estos países evoluciona de manera independiente del déficit: aunque éste se haya estabilizado, la deuda pública sigue creciendo a un ritmo que supera el del crecimiento de aquél. Es más: en un escenario de un déficit público estabilizado casi por completo, la deuda pública europea se triplicó. La conclusión que se extrae de ello es que la deuda pública de los Estados no es una consecuencia del déficit fiscal, sino que se desen- 
vuelve en forma autónoma, por lo que reducir el déficit fiscal no significa en forma alguna una reducción del endeudamiento del Estado. Aunque exceda el objeto de este artículo, el hecho de que la deuda pública crezca a un ritmo semejante al producto bruto sugiere que, antes que un modo de financiamiento del Estado, la deuda pública es una forma de participación en el ingreso nacional. En cualquier caso, si aceptamos que la recaudación tributaria tiene una relación con la deuda pública, otro corolario que se extrae de esos datos es que la estabilidad fiscal y la regla fiscal no hacen innecesarios los aumentos de tributos, lo cual es, como vimos más arriba, uno de los presuntos atributos de la regla fiscal.

Veamos el caso de Estados Unidos, que a nivel federal ha carecido en todo momento de una regla fiscal y por lo tanto ha podido llevar a cabo una política fiscal activa.

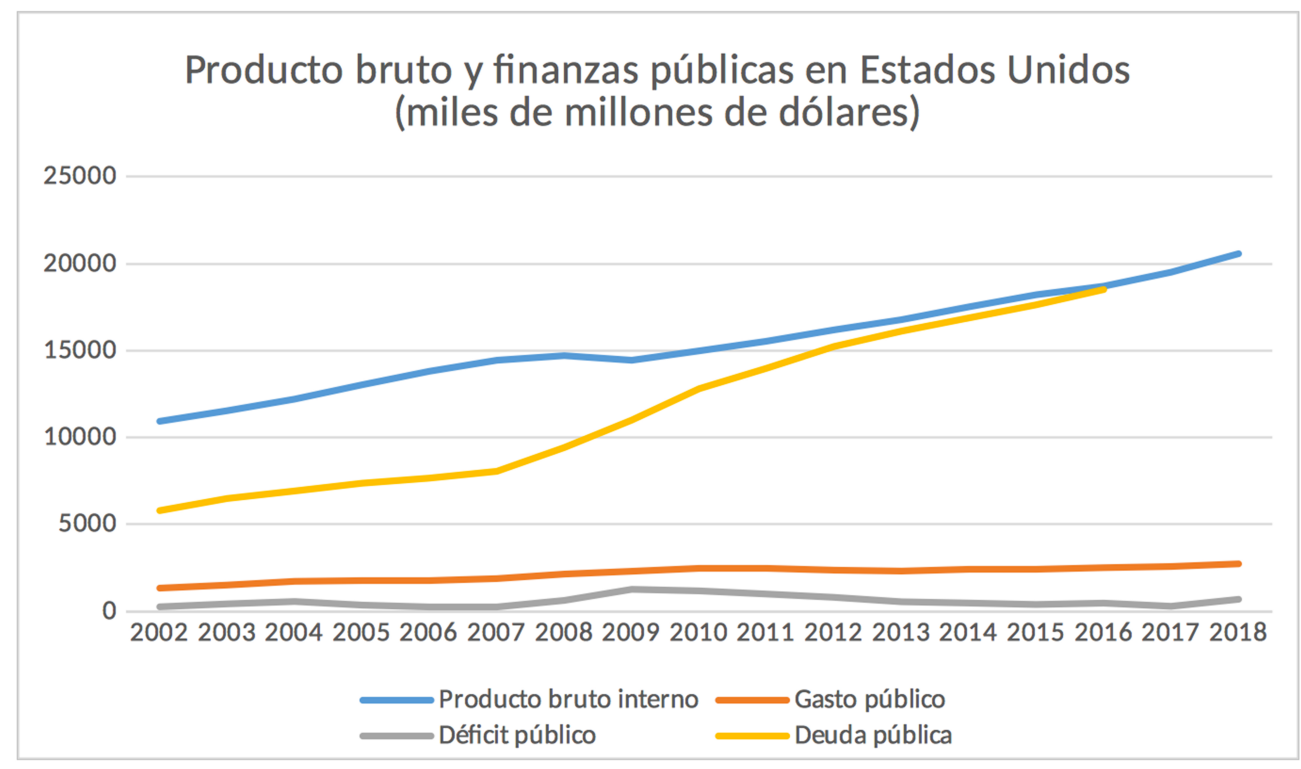

Fuente: Banco Mudial, https://datos.bancomundial.org/indicator/

La primera diferencia con el caso europeo, es que el gasto público sigue una evolución siempre ascendente, siquiera en forma leve, sin importar si se está o no en períodos de recesión. El déficit, por su parte, se eleva en los momentos de crisis (por ejemplo en 2008-2009), y se reduce en los auges, lo que obedece a las bajas y alzas -respectivamente- de la recaudación tributaria que son consecuencias naturales de la baja y alza del producto. En otras palabras, en Estados Unidos no hay una política de reducción y estabilización del déficit ni del gasto público, sino que el gobierno ha llevado a cabo una política fiscal moderadamente activa. 
Lo importante es que, en ese contexto, no dejan de existir crisis (lo que corresponde a la caída del producto de 2009), pero la recuperación es rápida y no se entra, como en Europa, en un período largo de estancamiento y crisis sucesivas. El único punto de coincidencia con Europa refuerza esa conclusión: el abatimiento más importante del déficit público en Estados Unidos, en 2008, es seguido de la única crisis importante del período.

En suma, otra vez nos encontramos con un escenario opuesto a las predicciones de la teoría neoclásica, y en especial contrario a las hipótesis que justifican la regla fiscal.

En cuanto a la deuda pública, se reitera, pero con mucha más contundencia, la observación realizada en Europa. En efecto, la evolución de la deuda pública de Estados Unidos muestra su completa independencia del déficit público, e incluso con la recaudación tributaria, lo cual ratifica que, en contra de la afirmación neoclásica de que la deuda pública es básicamente una forma de financiación del Estado, ella tiene causas independientes de los restantes elementos de las finanzas públicas.

Estos datos estadísticos no pasaron inadvertidos al mundo académico. Los autores poskeynesianos los han utilizado, como era previsible, como confirmación de sus hipótesis (Barba, 2006; Heimberger, 2017). Del otro lado, y sin considerar -por ser imposible su cita- los silencios, algunos antiguos defensores de la estabilidad fiscal y las reducciones del gasto público admitieron que el efecto positivo de dichas política es mucho más reducido que el que inicialmente pensaban (Perotti 2012), mientras que otros aceptaron que, al menos en el corto plazo, había que dar por cierta las hipótesis poskeynesianas en cuanto a los efectos positivos del aumento del gasto público, y recesivos de su reducción (Sutherland 1997). A mi entender, y de acuerdo con el criterio de Popper, es posible tener por refutadas las hipótesis neoclásicas acerca del gasto y el déficit públicos, principalmente las que nos dicen que las reducciones y restricciones al crecimiento del gasto público y el déficit tienen efectos económicos positivos. También podemos considerar refutada la hipótesis de que la deuda pública es una consecuencia del déficit fiscal. Aunque en la perspectiva de Popper no sea posible decir que las hipótesis poskeynesianas están confirmadas, puesto que ninguna hipótesis lo está nunca en forma definitiva, de todos modos ellas tienen un refuerzo importante puesto que, al menos en estos casos, sus pronósticos parecen confirmarse.

Vistas desde América Latina (y desde Uruguay en particular), estas experiencias, sin embargo, pueden ponerse al menos en discusión suponiendo que los efectos de las políticas fiscales de estabilización son diferentes en la periferia. Veamos 
la evolución de las mismas variables en Uruguay durante igual período, ya que, si bien carece de una regla fiscal en sentido jurídico, las relaciones entre su gasto público, su déficit y su producto pueden arrojar alguna luz al respecto.

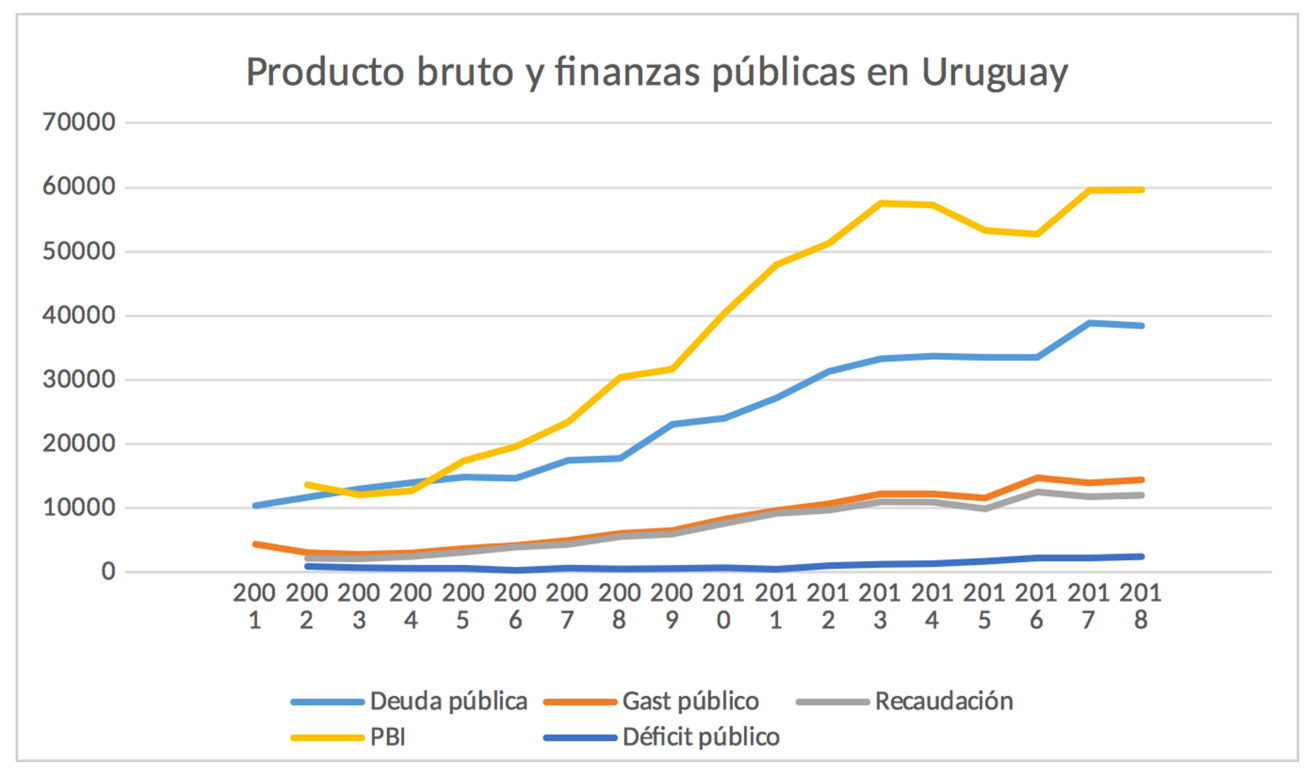

El gráfico muestra que desde 2003 Uruguay incrementó en forma sostenida su gasto público, con excepción de un período que coincide con una recesión en 2015 y 2016. El déficit fiscal, por su parte, fue poco importante hasta -aproximadamente- 2012, pero incluso a partir de esa fecha no fue de grandes dimensiones. Para ser más específicos, digamos que tanto el gasto público como la recaudación tributaria (que en este caso presento por separado) evolucionaron al mismo ritmo que el producto bruto: crecen y disminuyen toda vez que este último hace lo propio. Ello significa que los gobiernos uruguayos de este siglo no han seguido ni políticas fiscales de estabilización de corte neoclásico, porque no redujeron el gasto público en forma sostenida ni se propusieron una disminución del déficit, ni tampoco políticas fiscales activas de tipo poskeynesiano, porque no impulsaron ni mantuvieron el gasto público en momentos de recesión.

De cualquier manera, se puede extraer alguna conclusión teórica interesante. Los años de recesión en Uruguay fueron precedidos de varios años de aumentos sistemáticos del gasto público y el comienzo de la visibilidad del déficit. Esto no cumple con la predicción poskeynesiana, a diferencia -según vimos más arriba- de lo que sucedió en fechas similares en Europa y Estados Unidos. Esa circunstancia 
es la que, como señalé más arriba, me hace pensar que las hipótesis poskeynesianas tienen una enorme salvedad cuando las contrasta con las experiencias de países que, como Uruguay y otros de América Latina, tienen estructuras productivas diferentes a los países centrales. Ello no quiere decir que la demanda no sea el único factor determinante de la evolución del producto, y que el gasto público no sea un elemento positivo de ella; ambas hipótesis son en general correctas también en nuestra región. Sin embargo, y si bien es un punto ajeno a este trabajo, entiendo que América Latina y el resto de los países periféricos tienen estructuras productivas débiles, altamente sensibles a la demanda exterior y a ciclos del capital también exteriores, con lo cual, a diferencia de los países centrales, es mucho más limitado el papel del gasto público como sustituto de la demanda privada. Claro que tampoco está confirmada la hipótesis neoclásica de que la reducción del gasto público tiene efectos positivos en el crecimiento económico, y que la elevación del gasto y la deuda pública tienen efectos negativos sobre el consumo y la inversión: Uruguay vivió muchos años de incremento del gasto y la deuda, y la mayor parte de ese período fue de crecimiento del producto.

Pero volvamos a nuestro objetivo inicial. La conclusión que se extrae de la información reseñada es que las hipótesis teóricas y predicciones que apoyan la regla fiscal, a saber que la contención del gasto público y el déficit evitan las crisis y contribuyen a mejorar el crecimiento económico, están refutadas. Los partidarios de dichas hipótesis podrían agregar (como vimos que hacen algunos) que las mismas se mantienen, pero menguadas en su fuerza, y que las recesiones (que las reducciones y contenciones del gasto público no lograron evitar) se deben a la incidencia de otros factores ajenos a la economía estatal. Ante esa versión moderada de las hipótesis se podría responder, en primer lugar, que precisamente la virtud de las medidas de control del incremento del gasto público radica en -digámoslo así- "dejar que el mercado haga lo suyo", porque las distorsiones se deben, supuestamente, a la intervención del Estado. Si las recesiones no obedecen a esa causa, entonces todo el entramado teórico neoclásico deja de ser aceptable.

La conclusión anterior es una aplicación para el caso concreto de la estrategia de validación de Popper, a la que hice mención al inicio del apartado. Ella exige a las hipótesis teóricas hacer predicciones lo más precisas posibles, por lo que cuanto más vaga sea la hipótesis, más débil será, y cuando más compatible sea con observaciones contradictorias, menos confianza debemos tener en la misma. 
También está refutada la hipótesis de que el incremento de la deuda pública se contiene restringiendo el gasto y el déficit públicos, ya que aquella no guarda relación con este último sino que obedece a otros motivos, probablemente ajenos a las finanzas públicas. De esta forma queda también refutada la hipótesis del "compromiso de las generaciones futuras” a favor de la regla fiscal (Risso Ferrand, 2020, 17-18), ya que si bien puede admitirse que el aumento de la deuda pública compromete la política fiscal y económica futura, ello no es una consecuencia del déficit fiscal, sino de que la deuda pública tiene una dinámica de crecimiento autónoma del resto de los factores constitutivos de las finanzas públicas.

Por lo tanto, a mi entender corresponde rechazar los fundamentos teóricos para la consagración de una regla fiscal, sea cual fuere su jerarquía. Al contrario: en la medida en que, al menos en parte el gasto público ha mostrado ser una herramienta relevante más contra las recesiones, no es recomendable que los Estados restrinjan de antemano el uso de dicha herramienta mediante disposiciones internacionales o nacionales, de rango constitucional o legal.

\section{Las reglas fiscales desde el punto de vista del diseño político}

El fundamento más profundo para sostener normativamente la regla fiscal es una prolongación de la concepción de la teoría neoclásica sobre la institucionalidad fiscal, concepción que ya es más que centenaria.

En su versión más antigua, esta concepción parte del supuesto general, ya visto más arriba, de que el gasto público y los tributos son factores distorsionantes que deben restringirse todo cuanto sea posible. Luego se nos dice que el parlamento puede ser visto como una reunión de representantes de personas que viven en la sociedad, y que, en su conjunto, refleja la suma de las utilidades individuales de todos los votantes. En este punto se introduce en el análisis una noción que todavía no hemos tocado, pero que es esencial en la estructura teórica neoclásica: el “óptimo de Pareto”. Este es un estado en el que nadie puede mejorar su posición sin empeorar la del resto, y que, en la teoría neoclásica, se cumple cabalmente sólo en una economía sin interferencias del Estado ni otros agentes colectivos (Pareto, 1945, 266 y ss.). Trasladando ese concepto al parlamento, esta concepción afirma que un modelo de decisión óptimo es aquel en el que la regla es la unanimidad, porque sólo de esa forma se contemplan todas las utilidades de cada uno y se asegura que ninguno de los votantes se vea perjudicado. No obstante, y asumiendo que la unanimidad es difícilmente aceptable como regla de decisión, se propone una mayoría muy elevada (generalmente 5/6, que fue 
originalmente la propuesta de Wicksell) como requisito para aprobar un nuevo gasto público y para crear tributos (Wicksell, 1994).

Si bien en esta versión del constitucionalismo fiscal neoclásico desemboca en reglas sobre mayorías, nos sirve para revelar la idea fundamental que también inspira a la regla fiscal: cuando el gasto público y los tributos están en manos de un parlamento democrático, se corre el riesgo de que la mayoría decida aumentarlos, lo cual no es deseable por su carácter distorsivo. Por lo tanto, es preciso, para estar teoría, establecer límites fuertes para el accionar de ese parlamento en materia de gasto público y tributos. Vistas así las cosas, tanto las mayorías elevadísimas (cercanas a la unanimidad) y la regla fiscal son variantes instrumentales de una misma idea madre.

Las versiones más modernas del constitucionalismo fiscal neoclásico sacan de escena el óptimo de Pareto, y colocan en su lugar otros conceptos, como la falta de “internalización de los costos” del gasto público, fruto de la "fragmentación” de puntos de vista en los parlamentos democráticos. Dicha fragmentación política es el fruto de la pluralidad social, étnica, religiosa, ideológica, etc., de la sociedad contemporánea, y lleva a que los "grupos mayoritarios” aumenten el gasto público para favorecerse, descargando la carga fiscal en los "grupos minoritarios" (Kantopoulos \& Perotti, 1999; Von Hagen, 2002, 263-265).

Veamos las objeciones a estas hipótesis. En primer lugar, los referentes de esta teoría no presentan ninguna evidencia fáctica de que las mayorías parlamentarias de los países democráticos distribuyan las cargas fiscales de un modo desigual, y particularmente que la carga tributaria afecte a las minorías. Si por "minoría” se entiende a los empresarios, lo cual parece ser el subtexto de esa expresión utilizada para los neoclásicos, su tesis sería cierta si fácticamente la carga tributaria recayera en su mayor porción sobre dichas personas. Pero ello no es lo que sucede. En el caso de Uruguay, por lo pronto, es razonable conjeturar que la mayor parte de la carga económica de los impuestos nacionales recae, directamente o a través de la traslación, en los trabajadores y jubilados (Blanco 2012), por lo que se estaría ante un supuesto fáctico exactamente opuesto al propuesto por esta corriente. Dado que casi todos los países tienen, en su mayoría, una estructura tributaria semejante, probablemente sea ese el escenario más generalizado.

En el terreno estrictamente normativo, los argumentos contrarios a las tesis del constitucionalismo fiscal neoclásico derivan de los fundamentos de la democracia deliberativa, entendiendo por tal la primacía que tienen decisiones de un órgano co- 
lectivo, elegido en forma libre e irrestricta, y que decide por mayoría luego de una deliberación pública lo más amplia posible. En primer lugar, el enfoque neoclásico de la Constitución supone que la mayoría parlamentaria es igual a la mayoría numérica en la sociedad civil, lo cual dista de ser cierto. La investidura democrática de los parlamentarios no implica una presencia de ciertos grupos sociales como actores políticos, sino en todo caso una probabilidad de que sean tenidas en cuenta las pretensiones de dichos grupos. Por lo tanto, una mayoría parlamentaria es, como regla, sólo eso: una mayoría de parlamentarios. Desde luego que esa mayoría está respaldada a través del voto por una mayoría de personas votantes, pero ella no es un reflejo de la composición social de los intereses o pretensiones sostenidos por los parlamentarios.

Si la objeción se mantiene como una mayor proclividad a la permeabilidad del voto en el parlamento por las pretensiones de los grupos sociales, vehiculizadas a través de diversas formas de presión, eso es uno de los grandes méritos de la democracia, no un defecto a corregir. Al contrario de lo sostenido por el constitucionalismo fiscal neoclásico, este rasgo es favorable a una mayor imparcialidad en las decisiones públicas, dado que muchas pretensiones y puntos de vista opuestos tienen que tenerse en cuenta, lo cual va a favor de la atención a todos los grupos sociales y no a un trato desigual (Gargarella, 1997, 261-262).

Esas consideraciones son totalmente aplicables al gasto público y a los tributos. Tanto el nivel general del gasto público, como también su distribución, y la determinación de la estructura tributaria y el nivel de recaudación, son una parte esencial de las políticas públicas. Por esa razón, la representatividad, la pluralidad y la posibilidad efectiva de atender a los afectados, no sólo son rasgos aplicables al gasto público y los tributos, sino que lo es quizás en mayor medida que en otras áreas. En ese sentido, es tan atendible la pretensión de aumentar el gasto público -por ejemplo- de los usuarios de la salud pública, como la de los empleados públicos también de aumentarlo, como la de ciertos grupos de empresarios que solicitan un beneficio tributario. El único motivo por el que el gasto público fuera restringible a priori mediante -por ejemplo- una regla fiscal supranacional o constitucional, es que tuviera algún rasgo intrínsecamente negativo que requiriera dejarlo fuera de la decisión legislativa. Pero, en ese caso, volveríamos a la discusión de los apartados anteriores acerca de las tesis teóricas sobre el mismo. Si se aceptan las conclusiones propuestas en esos apartados, el hecho de que el gasto público no tenga connotaciones negativas, sino que, al contrario, sea un factor necesario y positivo en la política económica, desaparece una objeción para su limitación. 
Por otro lado, el constitucionalismo fiscal neoclásico es objetable en el plano epistemológico. La adopción constitucional o supranacional, o incluso legal, de una solución basada en una concepción teórica en desmedro de otras (que, como vimos, existen y disputan con la teoría neoclásica), supone que la teoría elegida es indefectiblemente preferible a esas otras que se dejan de lado. Eso de por sí es un grave error epistemológico: como vimos con Popper, las disputas teóricas no se dirimen mediante Constituciones, Tratados o leyes, sino con evidencias empíricas. Pero incluso si nos ciñéramos a la faz política del gasto público, siempre es preferible que una decisión no quede petrificada, sino que se resuelva en forma diferente según el criterio de la mayoría. Esto, que se ha denominado "superioridad epistemológica" de la democracia (Nino, 1989, 124 y ss.), es una consecuencia de la imposibilidad de trasladar al terreno normativo cualquier criterio de validación propio de las descripciones, como por ejemplo el de Popper que se mencionó en este trabajo. Dada esta circunstancia, la única corrección posible es la que surge de una decisión tomada por los involucrados, previa deliberación. En materia de gasto público, ello quiere decir que, en términos políticos, lo único que puede llamarse correcto es lo que decida un órgano electivo y plural que debata en forma pública. Desde ese punto de vista, cualquier restricción, como por ejemplo la regla fiscal, implica un déficit epistemológico ya que aleja a esa sociedad -o conjunto de sociedades- de aquel objetivo. 


\section{Referencias}

Alesina, A. y Perotti, R. (1994). The political economy of budget deficit, National Bureau for Economic Research (NBER) Working papers, Nro. 4637. https:// www.nber.org/papers

Arestis, P. y Sawyer, M. (2004). On Fiscal Policy and Fiscal Deficit. Intervention. Journal of Economics, 1(2).

Arrow, K. (1974). General economic equilibrium: Purpose, analytic techniques, collective choice. The American Economic Review, 64(3).

Barba, A. (2006). The Ebb and Flow of Fiscal Activism. Contributions to Political Economy, 25, Oxford University Press.

Barro, R. (1981). Output effects of government purchases. Journal of Political Economy, 89(6).

Bell, S. (2001). The role of the State and the Hierarchy of money. Cambridge Journal of Economics, 25.

Bertola, G. y Drazen, A. (1991). Trigger points and budget cuts: explaining the effects of fiscal austerity. NBER Working Papers, Nro. 3844. https://www.nber.org/ papers

Blanco, A. (2012). Efectos de los tributos. Revista Tributaria. 226.

Blanco, A. (2020). Las reglas fiscales y la Constitución uruguaya. Revista de la Facultad de Derecho, (49).

Courtis, C. (2006). El juego de los juristas. Ensayo de caracterización de la investigación dogmática. AA.VV.: Observar la ley. Ensayos sobre metodología de la investigación jurídica (edición de Christian Courtis).

Fanelli, J. P. (2011). Reglas fiscales, ciclo y volatilidad económica. Revista de Economía Política de Buenos Aires, 5, 9-10. 
Gargarella, R. (1997). Full representation, deliberation and impartiality. AA.VV.: Deliberative democracy (edited by Jon Elster).

Giavazzi, F. y Pagano, M. (1990). Can severe fiscal contractions be expansionary? Tales of two small European countries. NBER Working Papers, Nro. 3372. https://www.nber.org/papers

Heimberger, P. (2017). Did fiscal consolidation cause the double-dip recession in the euro area? Review of Keynesian Economics, 5(3).

Kalecki, M. (1971). Selected Essays on the Dynamics of Capitalist Economy, Cambridge University Press, Cambridge (Reino Unido). Hay edición española: Ensayos escogidos sobre dinámica de la economía capitalista.

Kantopoulos, Y. y Perotti, R. (1999). Government Fragmentation and Fiscal Policy Outcomes. Evidence from OECD Countries. AA.VV.: Fiscal Institutions and Fiscal Performance (James Poterba editor).

Keynes, J. M. (2018). General Theory of Employment, Interest and Money, PalgraveMacmillan, Cambridge (Reino Unido). Hay edición española: Teoría general de la ocupación, el interés y el dinero.

Kopits, G. y Symansky, S. (1998). Fiscal Policy Rules, Occasional Papers Nro. 162, Fondo Monetario Internacional. https://www.elibrary.imf.org/

Larch, M. y Jonung, L. (2014). The fiscal stability and growth pact of the European Union. The New Palgrave Dictionary of Economics, Online Edition, Edited by Steven N. Durlauf and Lwrence E. Blume. http://www.researchgate.net/ publication/263734505

Lucas Jr, R. (1975). An equilibrium model of the business cycle. Journal of Political Economy, 83(6)

Morales Rossi, M. y Posada Leguisamo, M. del P. (2010). Regla Fiscal. Aplicación al caso uruguayo. Monografía presentada ante la Facultad de Ciencias Económicas de la UDELAR. https://www.colibri.udelar.edu.uy/jspui/handle/ 20.500.12008/332 
Nino, C. S. (1989). El constructivismo ético, Centro de Estudios Constitucionales.

Nino, C. S. (1993). Derecho, Moral, Política. Doxa. Cuadernos de Filosofía del Derecho, (14).

Pareto, V. (1945). Manual de economía política.

Parguez, A. (2000). A monetary theory of Publica Finance. http://www.neties.com/ presentation\%20ap.html

Perotti, R. (2012). The “Austerity Myth”. Gain without Pain? AA.VV.: Fiscal Policy after the Financial Crisis (A. Alesina and F. Giavazzi-editors).

Popper, K. R. (1994). Conjeturas y refutaciones.

Risso Ferrand, M. (2020). Nuevas cláusulas fiscales en la Constitución. El combate al déficit fiscal y el endeudamiento. Revista de la Facultad de Derecho, (49).

Sarlo, O. (2006). El marco teórico de la investigación dogmática. AA.VV.: Observar la ley. Ensayos sobre metodología de la investigación jurídica (ed. de Christian Courtis).

Sen, A. (1999). Sobre ética y economía.

Simon, H. (1959). A Behavioral Model of Rational Choice. Quarterly Journal of Economics, 69, Oxford.

Sutherland, A. (1997). Fiscal crises and aggregate demand: Can high public debt reverse the effects of fiscal policy? Journal of Public Economics, 65(2),

Von Hagen, J. (2002). Fiscal Rules, Fiscal Institutions and Fiscal Performance. Economic and Social Review, 33(3).

Walras, L. (1987). Elementos de economía política pura o teoría de la riqueza social.

Wicksell, K. (1994). A new principle of just taxation. En AA.VV.: Classics in Theory of Public Finance (edited by Richard Musgrave and Alan Peacock). 


\section{Notas}

${ }^{1}$ Indiquemos brevemente que el Producto bruto, o Producto Bruto Interno (PBI), es la suma del valor monetario (es decir, expresado en dinero), de todos los bienes y servicios que se producen en un país o una región, durante un período dado (un año civil, un período interanual, etc.).

${ }^{2}$ La literatura neoclásica que fundamenta la restricción y el recorte del gasto público es vastísima. Me limito a citar tres trabajos que, además de ser referencia frecuente en la materia, desarrollan en profundidad los argumentos e intentan apoyarlos en evidencias empíricas.

${ }^{3}$ De la misma manera que con la literatura neoclásica referida a los efectos de las reducciones del gasto público, sólo cito en la ocasión un par de artículos donde se expone claramente la propuesta, para no abrumar al lector con referencias que seguramente serán incompletas.

${ }^{4}$ Tanto en esta como las gráficas sucesivas, los años no cubiertos por alguna de las líneas corresponden a aquellos en los que la fuente consultada no arroja información de la variable de que se trate.

${ }^{5} \mathrm{Si}$ bien este artículo es anterior a la crisis, anticipa esa admisión parcial de los efectos positivos del gasto público.

${ }^{6}$ Aunque quede fuera del objeto del trabajo, advierta el lector que esta recesión no fue registrada por los organismos estadísticos uruguayos, que no informan de un fenómeno de ese tipo en el período indicado. Sin perjuicio de otras connotaciones, esto es una fuerte alerta respecto de confiabilidad de las informaciones estadísticas, ya que la utilización de diferentes criterios de medición (por ejemplo, "moneda constante” o "dólares a precios actuales") puede terminar en resultados completamente diferentes. 